\title{
Characterizing Building Blocks of Resource Constrained Biological Networks
}

\author{
Yuanfang Ren ${ }^{1, \dagger}$, Ahmet Ay $^{2, \dagger}$, Alin Dobra $^{1}$, Tamer Kahveci ${ }^{1, *}$ \\ ${ }^{1}$ Computer and Information Science and Engineering, University of Florida, Gainesville, FL 32608 \\ yuanfang/adobra/tamer@cise.ufl.edu \\ ${ }^{2}$ Departments of Biology and Mathematics, Colgate University, Hamilton, NY 13346 \\ aay@colgate.edu \\ * Corresponding author, † Equal contribution
}

\begin{abstract}
Identification of motifs-recurrent and statistically significant patternsin biological networks is the key to understand the design principles, and to infer governing mechanisms of biological systems. This, however, is a computationally challenging task. This task is further complicated as biological interactions depend on limited resources, i.e., a reaction takes place if the reactant molecule concentrations are above a certain threshold level. This biochemical property implies that network edges can participate in a limited number of motifs simultaneously. Existing motif counting methods ignore this problem. This simplification often leads to inaccurate motif counts (over- or under-estimates), and thus, wrong biological interpretations. In this paper, we develop a novel motif counting algorithm, Partially Overlapping MOtif Counting (POMOC), that considers capacity levels for all interactions in counting motifs. Our experiments on real and synthetic networks demonstrate that motif count using the POMOC method significantly differs from the existing motif counting approaches, and our method extends to large-scale biological networks in practical time.
\end{abstract}

\section{CCS CONCEPTS}

- Applied computing $\rightarrow$ Biological networks; Bioinformatics; Systems biology;

\section{KEYWORDS}

Motif counting; partial overlap; reaction capacity; biological networks

\section{INTRODUCTION}

One of the most fundamental challenges in computational network studies is the motif counting problem. A network motif is a pattern of local interconnections (i.e., a small subnetwork) observed significantly more frequently in a given network than in a random network of the same size [1]. Existing studies have already uncovered existence of network motifs such as feed forward loop and bifan [6]. Motifs utilize the basic control mechanisms to govern biologically important dynamic behaviors, such as oscillations, generation of molecular pulses, and rapid or delayed responses $[1,11]$. Thus, the presence or relative abundance of motifs in biological networks is often used to characterize their topology, function, and robustness [7]. Network motifs have been effectively used to study

Permission to make digital or hard copies of part or all of this work for personal or classroom use is granted without fee provided that copies are not made or distributed for profit or commercial advantage and that copies bear this notice and the full citation on the first page. Copyrights for third-party components of this work must be honored For all other uses, contact the owner/author(s).

ACM-BCB'18, August 29-September 1, 2018, Washington, DC, USA

(c) 2018 Copyright held by the owner/author(s)

ACM ISBN 978-1-4503-5794-4/18/08.

https://doi.org/10.1145/3233547.3233680
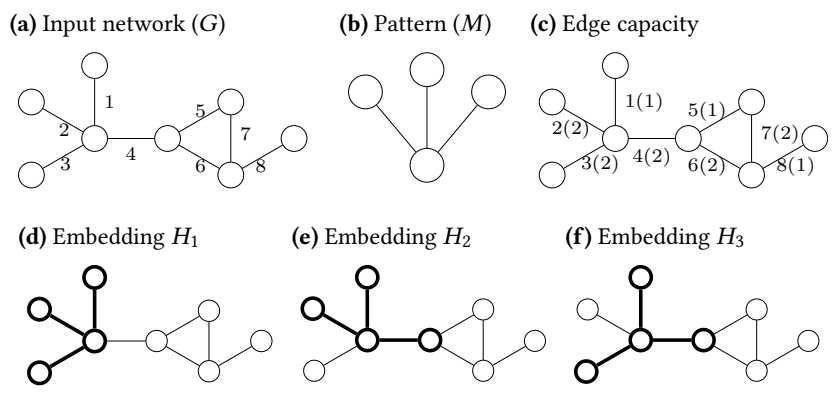

(e) Embedding $\mathrm{H}_{2}$

\section{(f) Embedding $H_{3}$}

(g) Embedding $H_{4}$
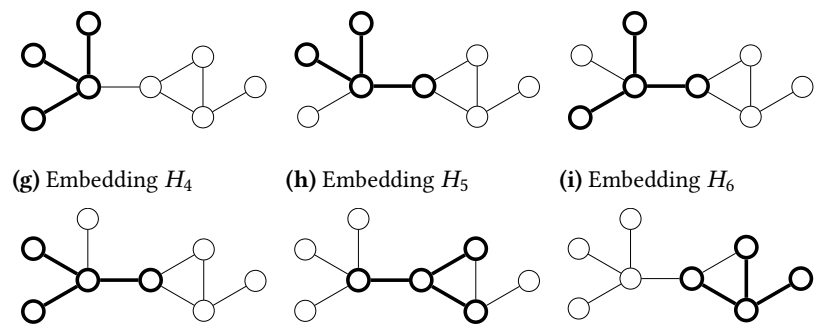

(h) Embedding $H_{5}$

(i) Embedding $H_{6}$
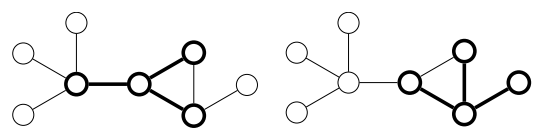

Figure 1: (a) A network with eight nodes and eight edges. (b) A motif pattern. (c) A network with edge capacities. $x(y)$ denotes the edge $x$ has the capacity $y$. (d) - (i) six motif embeddings in the network.

the biological processes that regulate transcription and to find the genetic factors that impact various diseases $[3,6]$.

Identifying motifs and counting them in biological networks is a computationally challenging task as it requires solving the subgraph isomorphism problem, which is NP-complete [2]. Several methods have been developed to count instances of a motif in a given network $[4,9]$. These methods could be categorized into two classes [4]. The first class counts all instances of a given motif ignoring the fact that some motifs may share edges ( $F_{1}$ measure) [9]. The second class counts all non-overlapping instances of a given motif, i.e., those which do not share any edge ( $F_{2}$ measure). Figure 1 shows the difference between these two frequency measures on a hypothetical network $G$. Consider the motif pattern $M$ in Figure $1 \mathrm{~b}$. Our input network $G$ in Figure 1a yields six possible embeddings of $M$ shown in Figures 1d-1i. Thus, $F_{1}$ measure of $M$ in $G$ is six. However, out of these six embeddings at most two can be chosen without picking the same edge multiple times (e.g., Figures $1 \mathrm{~d}$ and 1i). Thus, $F_{2}$ measure of $M$ in $G$ is two.

Notice that both $F_{1}$ and $F_{2}$ measures make opposite, yet very strong assumptions regarding how the cells realize interactions. The former one assumes that the same interaction can participate in an arbitrary number of motif instances at the same time. The latter one limits the participation of an interaction to a single motif instance. Although, these two assumptions simplify the motif counting problem, they rarely reflect how cells operate interactions. Each interaction utilizes the molecules participating in that interaction. Thus the abundance of the interacting molecules makes it 
possible to include the corresponding edges appear in multiple (yet limited) number of motif instances. For example, depending on the concentration level of a metabolite, an enzymatic reaction may take place in one (low reaction/edge capacity) or many metabolic pathways (high edge capacity) [12]. We use the term capacity of an edge to denote the number of motif instances that edge can participate simultaneously. Thus, even if two cells have the same underlying biological network topology, they may yield different number of motifs of the same topology. For example, if we allow partial overlap of the motif $M$ in the network $G$ (see Figures $1 \mathrm{~b}$ and 1c), we find four possible embeddings of motif $M$ in $G$ (Figures $1 \mathrm{~d}, 1 \mathrm{~g}, 1 \mathrm{~h}$ and 1i). A motif counting approach that ignores the edge capacities will lead to unrealistic motif counts, and wrong biological interpretations. Motif counting approaches that take edge capacities into account are needed.

\section{METHODS}

In this study, we build a new motif counting algorithm that allows partial overlap between different embeddings of a given motif on the target network. Briefly, given a target network, a motif topology, and a positive capacity value for each interaction in the target network, we count the maximum number of ways to place the motif on the target network, so that no edge appears in more motif embeddings than its capacity. Notice that the classical counting measures $F_{1}$ and $F_{2}$ are special instances of our measure $[5,10]$. If we set the capacity of all edges to one, our motif counting problem reduces to non-overlapping motif counting with $F_{2}$ measure. Similarly, if we set the edge capacities to infinity our problem reduces to motif counting using $F_{1}$ measure.

We develop a novel motif counting method called Partially Overlapping MOtif Counting (POMOC), that computes the number of partially overlapping instances of a given motif in a given network. $P O M O C$ first finds all instances of a given motif $M$ in the network $G$, then it chooses the motif instances that are guaranteed to exist without using any edge more than its capacity. For a given motif embedding, if the capacities of its edges are more than the number of embeddings those edges are part of, this embedding exists in our solution. Next, for the remaining motif instances, $P O M O C$ randomly adds some embeddings whose edges are not more than the capacity constraints, into the resulting embedding set. It gradually expands the resulting set by taking one embedding out of the resulting set and inserting another two embeddings to the set. For further details on our method see [8].

\section{RESULTS}

Our experiments on both synthetic and real networks demonstrate that motif count using POMOC significantly differs from the existing motif counting approaches, and our approach extends to large-scale biological networks in practical time. Our results show that $P O M O C$ finds substantially different number of motifs than that with $F_{1}$ and $F_{2}$ measures on a $S$. cerevisiae transcription regulatory network (see Table 1). We observe that there is a massive variation in the abundance of different motif topologies from only one up to over two thousand. There is only one instance of cascade. Thus, this motif instance occurs in all three measures as it has no other instance to overlap with. More importantly, partially overlapping motif counting varies greatly in the interval defined by $F_{1}$ and $F_{2}$ measures for different motif topologies. For instance, for biparallel, it is closer to $F_{1}$ measure, while it is closer to $F_{2}$ measure for bifan, and equally distant to $F_{1}$ and $F_{2}$ measures for feed forward loop. This demonstrates that the amount of overlap among different embeddings of a motif depends not only on the network topology but also the motif topology. Similar to Milo et al. (2002) [6], we find that bifan and feed forward motifs are the most abundant motif
Table 1: Average and standard deviation of the motif counts for four motif topologies using the $P O M O C$ method and, motif counting with $F_{1}$ and $F_{2}$ measures.

\begin{tabular}{lllll}
\hline & Feedforwad & Bifan & Biparallel & Cascade \\
\hline$F_{1}$ & 73 & 2026 & 11 & 1 \\
$F_{2}$ & 24 & 137 & 5 & 1 \\
POMOC & $54 \pm 0.5$ & $426.4 \pm 7$ & $10 \pm 0$ & $1 \pm 0$ \\
\hline
\end{tabular}

types in this network. These two motif topologies also show small variation across the $14 \mathrm{~S}$. cerevisiae transcription networks for the $P O M O C$ method. The running times for motif counting with $F_{1}$ and $F_{2}$ measures are less than 1 second in all real network experiments. POMOC method's running time-in average 22 seconds-is comparable to those of $F_{1}$ and $F_{2}$ measures. For a detailed description of our results see [8].

\section{CONCLUSIONS}

Motif counting in biological networks is an important tool to decipher the topology of biological networks and its function. Existing motif counting approaches either count all or non-overlapping instances of a given motif. This results in either over- or underestimation of the motif counts. In this paper, we presented a novel motif counting method, $P O M O C$, based on edge capacities of a given network. Since biological interactions are resource limited, leading to varying edge capacities, we hypothesize that our method provides a more accurate approach to counting network motifs in biological networks than existing methods. Although, the $P O$ $M O C$ method is slightly slower than motif counting with $F_{1}$ and $F_{2}$ measures, our method remains to be practical for all network sizes and motifs we test here. Our results on a $S$. cerevisiae transcriptional regulatory network suggest that oxidative stress is more disruptive to abundance and organization of network motifs than genetic mutations. Our analysis also suggests that by focusing on the edges that lead to variation in motif counts, our method can be used to find important genes, and to reveal subtle topological and functional differences of the biological networks under varying genetic backgrounds and growth conditions.

\section{REFERENCES}

[1] Uri Alon. 2007. Network motifs: theory and experimental approaches. Nature Reviews Genetics 8, 6 (2007), 450

[2] Michael R Garey and David S Johnson. 1979. Computers and intractability: A guide to the theory of npcompleteness (series of books in the mathematical sciences), ed. Computers and Intractability (1979), 340.

[3] Yanhui Hu and et al. 2011. An integrative approach to ortholog prediction for disease-focused and other functional studies. BMC Bioinformatics 12, 1 (2011), 357.

[4] Akihiro Inokuchi and et al. 2003. Complete mining of frequent patterns from graphs: Mining graph data. Machine Learning 50, 3 (2003), 321-354.

[5] Michihiro Kuramochi and George Karypis. 2005. Finding frequent patterns in a large sparse graph. Data Mining and Knowledge Discovery 11, 3 (2005), 243-271.

[6] Ron Milo and et al. 2002. Network motifs: simple building blocks of complex networks. Science 298, 5594 (2002), 824-827.

[7] Robert J Prill and et al. 2005. Dynamic properties of network motifs contribute to biological network organization. PLoS Biology 3, 11 (2005), e343.

[8] Yuanfang Ren and et al. 2018. Characterizing Building Blocks of Resource Constrained Biological Networks. bioRxiv (2018). https://doi.org/10.1101/352450 arXiv:https://www.biorxiv.org/content/early/2018/06/20/352450.full.pdf

[9] Falk Schreiber and Henning Schwöbbermeyer. 2005. Frequency concepts and pattern detection for the analysis of motifs in networks. In Transactions on Computational Systems Biology III. 89-104.

[10] Ngoc Hieu Tran and et al. 2013. Counting motifs in the human interactome. Nature Communications 4 (2013), 2241.

[11] John J Tyson and et al. 2003. Sniffers, buzzers, toggles and blinkers: dynamics of regulatory and signaling pathways in the cell. Current Opinion in Cell Biology 15, 2 (2003), 221-231.

[12] Cheng Yung-Chi and William H Prusoff. 1973. Relationship between the inhibition constant (Ki) and the concentration of inhibitor which causes 50 per cent inhibition (I50) of an enzymatic reaction. Biochemical Pharmacology 22, 23 (1973), 3099-3108. 